\title{
Zur Rolle der Privatversicherung beim Schutz vor Katastrophen
}

\section{Einführung}

Tritt eine Katastrophe ein und erreichen die ersten Berichte die Öffentlichkeit, so gelten das Interesse und die Anteilnahme zunächst den menschlichen Opfern. Die Größe der Katastrophe wird in der öffentlichen Wahrnehmung meist in erster Linie an der Zahl von Toten und Verletzten gemessen. Hinsichtlich dieser Personenschäden vermag die Privatversicherung, um deren Rolle beim Schutz vor Katastrophen es im Folgenden gehen soll, nur vergleichsweise wenig zu bewirken: Die Lebensversicherung kann Hinterbliebenen die wirtschaftlichen Einbußen zumindest teilweise ausgleichen, die etwa durch den Tod des Versorgers eintreten. Die Krankenversicherung gewährleistet die Finanzierung der Heilbehandlung, darüber hinaus ggf. auch eine bessere medizinische Betreuung (etwa durch Übernahme der Kosten für einen Rücktransport aus dem Katastrophengebiet). Unfallversicherung sowie Krankentagegeldversicherung, Krankenhaustagegeldversicherung und Berufsunfähigkeitsversicherung helfen, den durch eine Körperverletzung entstandenen finanziellen Mehrbedarf und den Ausfall des Erwerbseinkommens zu decken. All dies vermag zwar die Folgen der Katastrophe in gewisser Weise zu lindern, die eigentlichen, an Leib und Leben eingetretenen Verluste jedoch keineswegs zu kompensieren.

Ganz anders ist die Lage hinsichtlich der durch die Katastrophe verursachten Sachschäden. Auch sie können für die Betroffenen einschneidende persönliche Folgen haben: wirtschaftliche Existenzen sind ruiniert, Arbeitsplätze in Gefahr. All dies vermag die Sachversicherung abzuwenden, indem sie durch die Auszahlung der Versicherungssumme eine Wiederherstellung des status quo ante ermöglicht. Dabei sind praktisch alle Sparten der Sachversicherung angesprochen, von der Feuer- und der Gebäudeversicherung über die Hausratversicherung bis hin zur Ausfall- und zur Betriebsunterbrechungsversicherung. Freilich kann auch in diesen Fällen ein bestehender Versicherungsschutz nur die wirtschaftlichen Interessen und nicht etwa ein Affektionsinteresse decken.

Der Sachversicherungsschutz vor Katastrophenschäden wird international verbreitet nachgefragt, wenn auch mit deutlichen regionalen Abstufungen: So ist die Versicherung von Katastrophenrisiken in den westlichen Industriestaaten erheblich weiter verbreitet als etwa im asiatischen Raum. ${ }^{1}$ Insgesamt hat die Nachfrage dazu geführt, dass etwa im Jahr 2004 nahezu $40 \%$ aller weltweit durch Katastrophen verursachten Sachschäden durch eine Versicherung gedeckt waren. ${ }^{2}$

1 Eine (allgemeine) Statistik über das Verhältnis von Prämienaufkommen und Bruttoinlandsprodukt einzelner Länder findet sich in Sigma Nr. 2/2005, S. 17 u. 24 (abrufbar unter: www.swissre.com/sigma).

2 Sigma Nr. 1/2005, S. 3. 
Der Schutz vor Katastrophen, genauer gesagt: vor deren wirtschaftlichen Folgen, stellt die Privatversicherung freilich vor besondere Herausforderungen. Sie hängen insbesondere mit zwei charakteristischen Merkmalen von Katastrophen zusammen, nämlich mit der schwierigen Prognostizierbarkeit des Schadenseintritts und mit der drohenden exorbitanten Schadenshöhe. ${ }^{3}$ Beides erschwert die Kalkulation des Risikos und der davon abhängigen Prämie.

Im Folgenden sollen nach einem Überblick über wichtige Grundlagen (II) zunächst die Möglichkeiten betrachtet werden, die dem Versicherer für die Verbesserung der Kalkulierbarkeit von Katastrophenrisiken zur Verfügung stehen (III). Sodann werden die Wege aufgezeigt, die der einzelne Versicherer beschreiten kann, um die von ihm versicherten Katastrophenrisiken zu reduzieren (unten IV) und um sie ganz oder teilweise auf Dritte zu verlagern und auf diese Weise seine eigene Leistungsfähigkeit zu sichern (V). Anschließend ist der Umfang des Versicherungsschutzes für die einzelnen Katastrophenrisiken zu betrachten, wobei das Augenmerk insbesondere auf die aktuellen Themen Überschwemmungen und Terrorakte gerichtet werden soll (VI). Am Ende des Beitrags wird ein Fazit gezogen (VII).

\section{Grundlagen}

\section{Privatversicherung als privatautonome Risikovorsorge}

Versicherung ermöglicht Planungssicherheit. Der Versicherungsnehmer tauscht gleichsam das für ihn unwägbare Risiko künftiger wirtschaftlicher Belastung teilweise oder vollständig gegen eine feststehende Prämienlast ein. Durch die Bildung eines Kollektivs gleichartiger Risiken wird das Risiko des Einzelnen vergemeinschaftet; viele Versicherungsnehmer tragen durch Leistung der vereinbarten Prämie dazu bei, dass einige von ihnen, bei denen sich das Risiko verwirklicht, einen Ausgleich erlangen.

In der Privatversicherung beruht diese Solidargemeinschaft freilich nicht auf sozial motivierter staatlicher Anordnung, sondern grundsätzlich - sieht man einmal von Einschränkungen durch gesetzliche Versicherungspflichten ab - auf einer privatautonomen Entscheidung der Versicherungsnehmer und der Versicherer. Dies bedeutet, dass es sich nach den Regeln von Angebot und Nachfrage richtet, ob für ein ungewisses Ereignis, das einen Schaden hervorrufen kann, Versicherungsschutz besteht.

Generell lässt sich festhalten: Je schwieriger das Risiko kalkulierbar ist, umso eher wird es ungedeckt bleiben. Dass ein Risiko nicht versichert wird, kann unterschiedliche Gründe haben: Bisweilen fehlt es bereits an einem entsprechenden Angebot, da die Versicherer das Risiko wegen bestehender Kalkulationsschwierigkeiten als nicht versicherbar einstufen. Gibt es hingegen das Angebot eines Deckungsschutzes, so wird den kalkulatorischen Unwägbarkeiten regelmäßig bei der Prämienbemessung Rechnung getragen. Dies kann den Versicherungsschutz aus Sicht des Versicherungsnehmers unattraktiv erscheinen lassen, so dass er trotz vorhandener Möglichkeit von

3 Dahlke, VersR 2003, 25, 26. 
einer Deckung absieht. Nicht selten wird der Versicherungsnehmer aus denselben Gründen, die das Risiko für den Versicherer schwer kalkulierbar machen, auch die eigene Gefährdung als eher abstrakt empfinden und daher keinen Bedarf sehen.

Die auf beiden Seiten bestehende Entscheidungsfreiheit haben bereits die Motive zum Versicherungsvertragsgesetz, bezogen auf Risikoausschlüsse in der Feuerversicherung, mit folgenden Worten auf den Punkt gebracht: »Den Versicherungsunternehmen kann es nicht verwehrt werden, durch ihre Verträge die Haftung für eine Gefahr, die sie nicht übernehmen wollen, abzulehnen, und ebenso muss dem Versicherungsnehmer die Wahl frei bleiben, ob er dadurch, dass er auf eine Deckung gegen bestimmte Gefahren verzichtet, eine niedrigere Prämie oder durch Zahlung einer höheren Prämie die Deckung auch gegen jene Gefahren sich verschaffen will. ${ }^{4}$

\section{Schwierige Kalkulierbarkeit von Katastrophenrisiken}

Ein wesentlicher Faktor für die faktische Versicherbarkeit von Risiken besteht darin, dass die Schadenswahrscheinlichkeit im Voraus kalkulierbar ist. ${ }^{5}$ Dazu gehört neben der Wahrscheinlichkeit des Risikoeintritts insbesondere auch die voraussichtliche Schadenshöhe, einschließlich des gerade noch hinreichend wahrscheinlichen größtmöglichen Schadens (Probable Maximum Loss [PML]). Katastrophenrisiken zeichnen sich dadurch aus, dass sie typischerweise schwer kalkulierbar sind. Als Beispiel seien Wasserschäden angeführt: Es ist eine Erfahrungstatsache, dass regelmäßig Wohnungen durch den Betrieb von Waschmaschinen überflutet werden. Derartige Schäden kann der Versicherer statistisch erfassen; nach dem Gesetz der großen Zahl lässt sich aus der Schadenshäufigkeit in der Vergangenheit eine recht zuverlässige Prognose über die künftige Schadensentwicklung herleiten. Zudem kann der Versicherer zur Risikoreduzierung vergleichsweise präzise Schadensverhütungsmaßnahmen und Sorgfaltsregeln vereinbaren, da es sich um ein im Grundsatz beherrschbares, auf menschlichem Verhalten beruhendes Schadensrisiko handelt.

Ganz anders ist die Lage, wenn es sich bei der Überschwemmung um eine Naturkatastrophe handelt. So ist das »Jahrhunderthochwasser « von 2002 an der Elbe ein gänzlich ungewöhnliches Ereignis gewesen, das sich einer fundierten statistischen Regelbildung entzieht. Zugleich hat diese Flut nicht nur einzelne überschaubare Vermögenswerte betroffen, sondern in der Summe enorm hohe Schäden verursacht. Beide Faktoren, die bei derartigen Katastrophen zusammentreffen, erschweren - wie schon eingangs erwähnt - die Kalkulation: die schwierige oder gar unmögliche statistische Vorhersehbarkeit der Risikoverwirklichung und das ungewöhnlich große Ausmaß der Gesamtschadenshöhe.

Es gibt freilich auch Naturkatastrophen, die besser kalkuliert werden können als andere. So sind mittlerweile verbesserte Modelle verfügbar, mit denen sich die Häufigkeit

4 Motive zum Gesetz über den Versicherungsvertrag vom 30. Mai 1908, Nachdruck Berlin 1963, S. 155 (zu $\S \S 82$ bis 84).

5 Berliner, Gedanken zur Versicherbarkeit und zu Schiedsgerichtsklauseln, 1983, S. 3 ff.; ders., in: Farny (Hrsg.), Handwörterbuch der Versicherung, 1988, S. 951 ff. (Stichwort: Versicherbarkeit). 
und die Intensität von Stürmen prognostizieren lassen. ${ }^{6}$ Allerdings ermöglichen auch derartige Modelle regelmäßig nur eine grobe Einschätzung des künftigen Schadensrisikos.

Besondere Schwierigkeiten ergeben sich schließlich bei der Kalkulation von mit menschlichem Handeln zusammenhängenden Katastrophenrisiken. So wurde etwa vor den Anschlägen vom 11.9.2001 schon ein Flugzeugabsturz auf nur einen der Zwillingstürme des World Trade Center für so unwahrscheinlich gehalten, dass er in die versicherungsmathematische Berechnung des Risikos überhaupt nicht einfloss. ${ }^{7} \mathrm{Be}-$ ruht die Risikoverwirklichung nicht auf menschlichem Versagen, sondern - wie bei Terrorakten - auf einem planvollen Vorgehen, so kommt noch die Gefahr der Risikokumulation durch eine Kette von Anschlägen hinzu.

Die genannten, für Katastrophenrisiken charakteristischen Kalkulationsschwierigkeiten halten die Versicherungswirtschaft freilich keineswegs davon ab, derartige Risiken zu versichern. So sind beispielsweise im Jahr 2004 bei weltweit aufgetretenen Gesamtschäden von über 123 Mrd. USD durch 332 Katastrophen (fast ausschließlich Naturkatastrophen) insgesamt Sachschäden von 49 Mrd. USD versichert gewesen. ${ }^{8}$ Das entspricht einem Anteil von nahezu $40 \%$ am gesamten Schadensaufkommen durch Katastrophen.

In der langjährigen Schadensstatistik lässt sich trotz mancher Schwankungen insgesamt seit Ende der 1980er Jahre ein klarer Trend hin zu sehr hohen versicherten Katastrophenschäden ausmachen. Lag die weltweite Summe solcher Schäden von 1970 bis Mitte der 1980er Jahre vergleichsweise stabil bei unter 5 Mrd. USD (inflationsbereinigt zu Preisen von 2004) pro Jahr, so ist sie anschließend nicht mehr unter diese Schwelle gesunken, sondern vielmehr teils in zuvor unerreichte Höhen von $30 \mathrm{Mrd}$. USD und mehr pro Jahr gestiegen. ${ }^{9}$ Ausschlaggebend hierfür waren regelmäßig einzelne Großschadensereignisse wie Hurricanes (Andrew 1992, Ivan 2004), Taifune (Mireille 1991), Winterstürme (Lothar 1999), Erdbeben (Northridge 1994) sowie die Terroranschläge vom 11.9.2001. Die steigenden Schadensaufwendungen werden von den Versicherern, sofern es um Naturkatastrophen geht, nicht zwangsläufig in erster Linie auf die Klimaerwärmung zurückgeführt. ${ }^{10}$ Vielmehr werden dafür vor allem auch die steigende Wertekonzentration und Ansiedlungsdichte in gefährdeten Regionen angeführt, etwa in Florida, das in den letzten zwanzig Jahren ein starkes Bevölkerungs- und Wirtschaftswachstum aufweist. ${ }^{11}$ Als eine Ursache für die Steigerung der Schadensaufwendungen ist damit auch die erhöhte Schadensanfälligkeit auszumachen. Interessanterweise liegt ein Grund für die steigende Bevölkerungszahl und die damit verbundenen höheren Schadenaufwendungen gerade in dem angebotenen Versicherungsschutz, welcher in manchen Regionen staatlich subventioniert wird und somit Neuansiedlungen fördert. ${ }^{12}$

6 Sigma Nr. 1/2005, S. 13.

7 Wolgast, ifo-Schnelldienst 24/2001, S. 12; s. auch Münchener Rückversicherungs-Gesellschaft (Hrsg.), 11. September 2001, 2001, S. 8.

8 Sigma Nr. 1/2005, S. 3.

9 Sigma Nr. 1/2005, S. 6.

10 Sigma Nr. 1/2005, S. 13; weniger zurückhaltend noch Sigma Nr. 1/2004, S. 3.

11 Sigma Nr. 1/2005, S. 13.

12 Nell, Staatshaftung für Terrorrisiken, Working Papers on Risk and Insurance, Hamburg University, 2001, S. 3 (abrufbar unter http://www.rrz.uni-hamburg.de/IfVBL/WP_R\&I_4.pdf). 


\section{Versicherungstechnische Definition von Katastrophenrisiken}

Mit dem bereits angeführten Beispiel des Elbehochwassers von 2002 sind zugleich die wesentlichen Faktoren benannt, die den Begriff der Katastrophe prägen: Es handelt sich um ein überraschend eintretendes, nicht konkret vorhersehbares, auf Naturereignissen oder menschlicher Einwirkung beruhendes Ereignis, das außerordentlich hohe Schäden hervorruft. Diese Umschreibung ist ersichtlich mit gewissen Unschärfen verbunden. Es geht hier jedoch zunächst allein um eine phänomenologische Einordnung. Nach ihren Ursachen werden Katastrophen grob in Naturkatastrophen, die als durch Naturgewalten ausgelöste Ereignisse definiert werden, und sog. Man-made-Katastrophen (technische Katastrophen), die im Zusammenhang mit menschlichen Aktivitäten stehen, unterteilt. ${ }^{13}$

Präzisere Definitionen enthalten die von Versicherern erstellten Statistiken über Katastrophenschäden. So hat die von der Schweizerischen Rückversicherungs-Gesellschaft alljährlich erstellte Katastrophenbilanz für das Jahr 2004 als untere Schadensgrenzen in der Regel einen Versicherungsschaden von 37,5 Mio. USD oder einen Gesamtschaden von 74,9 Mio. USD angesetzt, bei Personenschäden eine Mindestzahl von 20 Toten oder Vermissten, 50 Verletzten oder 2.000 Obdachlosen. ${ }^{14}$

In den Versicherungsbedingungen, in denen die jeweiligen Katastrophenrisiken konkret aufgeführt werden (s. dazu unten VI), findet sich hingegen keine Definition der Katastrophe. Es würde im Rahmen des vertraglichen Regelwerks auch wenig Sinn machen, abstrakte Untergrenzen festzulegen, da der Versicherungsschutz regelmäßig ohnehin für die in Rede stehenden Risiken konkret festgelegt wird und insoweit (sieht man von Selbstbehalten ab) regelmäßig nicht eine Unter-, sondern die Obergrenze des Deckungsschutzes von entscheidender Bedeutung ist.

\section{Versicherungsrechtliche Regelungen zu Katastrophenrisiken}

Die Kategorien der Katastrophe oder des Katastrophenrisikos sind dem Versicherungsvertragsgesetz unbekannt. Allerdings befasst sich das Gesetz im Rahmen der Feuerversicherung mit bestimmten Katastrophenrisiken. Nach § 84 VVG haftet der Versicherer nicht, wenn der Brand oder die Explosion durch ein Erdbeben oder durch Maßregeln verursacht wird, die im Kriege oder nach Erklärung des Kriegszustandes von einem militärischen Befehlshaber angeordnet worden sind.

Bei dieser Regelung handelt es sich um einen gesetzlichen Risikoausschluss. Geht man den gesetzgeberischen Gründen hierfür nach, so findet sich in den Motiven lediglich der Hinweis, dass $§ 84$ VVG sich der herrschenden Praxis anschließe. ${ }^{15}$ In der Tat wurden Erdbeben- und Kriegsausschlussklauseln bereits im Jahr 1907, aus dem der Entwurf des am 30.5.1908 verabschiedeten Versicherungsvertragsgesetzes stammt, in der Versicherungswirtschaft verbreitet für geboten erachtet. ${ }^{16}$ Hintergrund hierfür war

13 Sigma Nr. 1/2005, S. 36. Nähere Differenzierung unten VI.

14 Sigma Nr. 1/2005, S. 37.

15 Motive, S. 157 (zu $\S 82$ bis 84).

16 Instruktiv hierzu der Beitrag von Röder in diesem Heft. 
wiederum die schwierige Kalkulierbarkeit für den Versicherer. ${ }^{17}$ Was insbesondere Erdbebenschäden angeht, so war die Assekuranz weltweit durch das große Erdbeben in San Francisco im Jahre 1906 alarmiert ${ }^{18}$.

Der gesetzliche Risikoausschluss ist nicht nur zu Gunsten, sondern auch zu Lasten des Versicherungsnehmers abänderbar. Die heutige Bedingungspraxis sieht verbreitet Erweiterungen des Risikoausschlusses vor. So werden häufig alle Schäden ausgeschlossen, die durch »Kriegsereignisse jeder Art« verursacht worden sind. Hinzu treten oft Risikoausschlüsse bezüglich innerer Unruhen oder Kernenergie (vgl. etwa § 1 Nr. 7 der Allgemeinen Bedingungen für die Feuerversicherung 1994 [AFB 94]).

Auf die sich aus derartigen Risikoausschlüssen ergebenden Folgen für den Versicherungsschutz für Katastrophenrisiken wird noch zurückzukommen sein (unten VI). Als Fazit hinsichtlich der versicherungsrechtlichen Vorgaben lässt sich zunächst festhalten, dass der deutsche Gesetzgeber keine gerade auf Katastrophenrisiken bezogenen zwingenden Vorgaben macht. Im Wesentlichen ist es daher Aufgabe der Vertragsparteien, Regelungen über Art und Umfang der zu deckenden Katastrophenrisiken zu treffen.

Diese Zurückhaltung des Gesetzgebers ist keineswegs selbstverständlich. Ein rechtsvergleichender Blick über die Grenzen erweist, dass insbesondere hinsichtlich Terroranschlägen, aber auch in Bezug auf Naturkatastrophen in anderen Staaten bisweilen weit reichende Vorgaben gelten. So ist es, um ein Beispiel zu nennen, nach französischem Recht untersagt, in bestimmten Sachversicherungsverträgen das Terrorrisiko auszuschließen; seine Deckung kann lediglich betragsmäßig beschränkt werden. ${ }^{19}$ Auch in Bezug auf Versicherungsschutz für Naturkatastrophen schränkt das französische Recht die Privatautonomie ein. ${ }^{20}$ Manche Rechtsordnungen wie etwa das US-amerikanische Recht sehen vor, dass der Versicherer zwingend die Einbeziehung von Terrorrisiken in den Deckungsumfang einer Sachversicherung anbieten muss, wobei es dem Versicherungsnehmer allerdings freigestellt ist, auf diesen Schutz im Interesse einer Prämienreduzierung zu verzichten. ${ }^{21}$ Das deutsche Recht setzt hingegen jedenfalls derzeit noch auf eine für beide Seiten, Versicherer wie Versicherungsnehmer, freiwillige Deckung von Terrorrisiken und bietet hierfür durch eine subsidiäre Staatsgarantie einen gewissen Anreiz (näher unten VI 3 b bb).

17 Johannsen/Johannsen, in: Bruck/Möller/Sieg/Johannsen, VVG, Bd. III (Feuerversicherung), 8. Aufl. 2002, Anm. H 33.

18 S. dazu wiederum den Beitrag von Röder in diesem Heft.

19 Art. L 126-2 CdA (Code des Assurances).

20 S. dazu Nell, Staatshaftung für Terrorrisiken (Fn. 12), S. 3 f.

21 Terrorism Risk Insurance Act of 2002, Pub. L No. 107-297, 116 Stat. 2322, 2324; s. dazu A. Bruns, JZ 2005, 13, 15 f. (Überblick); eingehend Manns, 112 Yale L.J. 2509, $2533 \mathrm{ff}$. (2003). 


\section{Möglichkeiten des Versicherers zur Verbesserung der Kalkulierbarkeit von Kata- strophenrisiken}

Unter den Kalkulationsgrundlagen des Versicherers sind, wie bereits erwähnt, hinsichtlich der Verwirklichung von Katastrophenrisiken zwei Faktoren besonders bedeutsam: zum einen die Wahrscheinlichkeit, mit der die Katastrophe eintreten wird, zum anderen Art und Umfang der durch ihren Eintritt verursachten Schäden. Was den ersten Faktor angeht, so bestehen insbesondere im Hinblick auf Naturkatastrophen Möglichkeiten, die Prognostizierbarkeit zu verbessern. $\mathrm{Zu}$ nennen ist vor allem die Klimaforschung, die sich mit Phänomenen wie der Entstehung von Wirbelstürmen, Flutwellen oder Erdbeben sowie insbesondere auch mit der globalen Erderwärmung ${ }^{22}$ befasst.

Bei unmittelbar auf menschliches Verhalten zurückzuführenden Katastrophen besteht hingegen eine vergleichbare Möglichkeit, die Vorhersehbarkeit des Katastropheneintritts zu verbessern, naturgemäß nicht. Dies gilt insbesondere für vorsätzliches Verhalten, wie es bei der Verwirklichung des Kriegs- und des Terrorrisikos (s. dazu noch unten VI 3) in Rede steht.

Die Kalkulierbarkeit lässt sich freilich außer durch naturwissenschaftliche und statistische Risikoanalysen auch dadurch verbessern, dass - unabhängig vom tatsächlichen Umfang des Risikos - der vertraglich vereinbarte Deckungsschutz begrenzt wird. Wichtigstes Instrument zur Steuerung des vom Versicherer übernommenen Risikos ist neben der Einschränkung auf bestimmte Schadensarten (Beispiel: gedeckt sind nur Sachschäden, keine Betriebsunterbrechungsschäden) die Festlegung der Versicherungssumme. Hinzu kommen weitere Vereinbarungen wie die Vereinbarung einer jährlichen Höchstentschädigungssumme, die unabhängig davon eingreift, wie häufig der Versicherungsfall binnen dieses Zeitraums eintritt. Freilich haben derartige Einschränkungen aus Sicht des Versicherungsnehmers den Nachteil, dass das Risiko zum Teil bei ihm verbleibt. Eine andere Möglichkeit zur Risikobegrenzung besteht darin, dass der Versicherer ein nach Eintritt des Versicherungsfalls mit kurzfristiger Wirkung ausübbares Kündigungsrecht vereinbart. Zwar muss dann der eingetretene Schaden noch vereinbarungsgemäß reguliert werden, der Versicherer kann jedoch anschließend die veränderte Risikosituation neu bewerten. ${ }^{23}$

\section{Versicherungstechnische Instrumente zur Verringerung der Schadenshöhe}

Lässt sich die Wahrscheinlichkeit des Eintritts einer Katastrophe präziser vorherbestimmen, so erleichtert dies zwar die Kalkulation, führt aber nicht zu einer Reduzierung des vom Versicherer zu tragenden Risikos. Eine solche Risikobegrenzung, die gleichfalls die Kalkulation erleichtert, steht dem Versicherer im Hinblick auf den zweiten Faktor, nämlich Art und Umfang der zu erwartenden Schäden, zu Gebote.

22 S. dazu Sigma Nr. 1/2004, S. $10 \mathrm{ff}$.

23 Wolgast, ifo-Schnelldienst 24/2001, 12, 13. 
Der Versicherer hat es nämlich in der Hand, die Gewährung von Versicherungsschutz davon abhängig zu machen, dass der Versicherungsnehmer bestimmte Schadensvorsorgemaßnahmen ergreift. Zwar werden die meisten Katastrophenschäden nicht durch menschliches Versagen herbeigeführt. So entfielen im Jahr 2004 von insgesamt 49 Mrd. USD an versicherten Schäden lediglich 3 Mrd. USD auf Schadensereignisse, die - im Unterschied zu Naturkatastrophen - in unmittelbarem Zusammenhang mit menschlichem Verhalten stehen, wie dies etwa bei Industrieunfällen und Flugzeugabstürzen regelmäßig der Fall ist. ${ }^{24}$ Damit ist es dem Versicherer für den größten Teil der versicherten Risiken nicht möglich, bereits den Eintritt der Katastrophe durch verhaltenssteuernde Regeln zu beeinflussen. Auch im Hinblick auf Naturkatastrophen ist jedoch eine Verhaltenssteuerung insofern möglich, als durch geeignete Vorkehrungen die Auswirkungen der Katastrophe auf den Eintritt und die Höhe des versicherten Schadens begrenzt werden können. Als Beispiele seien etwa genannt die erdbebensichere Errichtung eines Gebäudes, der Schutz vor Überschwemmungsschäden durch entsprechende technische Vorrichtungen oder der Einbau einer Sprinkleranlage.

Der Versicherungsnehmer kann zu derartigen Maßnahmen insbesondere dadurch motiviert werden, dass bereits der Abschluss des Versicherungsvertrages von ihrer Durchführung abhängig gemacht wird. Auch die Tarifgestaltung kann Anreize zur Schadensvorsorge schaffen, etwa indem bei einer bestimmten Bauweise des zu versichernden Gebäudes eine Prämienreduktion vorgesehen wird.

Zudem kann im Rahmen der gesetzlichen Vorgaben (s. insbesondere $\S 6 \mathrm{VVG}$ ) in den Versicherungsvertrag eine Klausel aufgenommen werden, derzufolge der Versicherungsnehmer Nachteile erleidet, insbesondere: den Deckungsschutz verliert, wenn die vereinbarten Maßnahmen aus vom Versicherungsnehmer zu vertretenden Gründen unterblieben sind und infolgedessen der Versicherungsfall eingetreten ist oder der Schaden sich vergrößert hat.

Der Versicherer hat mit den genannten Vorgehensweisen mehrere Möglichkeiten, die von ihm für sinnvoll erachteten Vorbeugungsmaßnahmen auch effektiv durchzusetzen. Voraussetzung ist eine sorgfältige Risikoabschätzung des Versicherers; sie wird in der Praxis nicht selten auch durch Versicherungsmakler oder durch Rückversicherer (s. noch sogleich V 2) durchgeführt.

\section{Möglichkeiten des Versicherers zur Verlagerung von Katastrophenrisiken}

Die soeben genannten Wege, die Kalkulierbarkeit des Risikos zu verbessern, ändern freilich nichts daran, dass Katastrophenrisiken im Grundsatz schwer kalkulierbar bleiben. Dies gilt insbesondere im Hinblick auf solche Katastrophen, die durch menschliches Verhalten hervorgerufen worden sind, wie Terroranschläge oder fahrlässig verursachte Unfälle in Chemie- oder Kernkraftwerken, aber auch für Naturkatastrophen wie Hurricanes, Erdbeben oder das schon erwähnte »Jahrhunderthochwasser« an der Elbe von 2002.

24 Sigma Nr. 1/2005, S. 3. 
Für einen bestimmten Zeitraum lässt sich die Schadenshöhe kaum auch nur näherungsweise prognostizieren. Dies beruht nicht zuletzt darauf, dass einige wenige Schadensereignisse jeweils einen Milliardenschaden verursachen können. Hinzu kommt, dass manche Schadensrisiken, insbesondere bei Naturkatastrophen, derart groß sind, dass sie die weltweite Gesamtkapazität der Versicherungswirtschaft übersteigen können. ${ }^{25}$

Stellt sich der Versicherer die Frage, ob er Versicherungsschutz für Katastrophenrisiken anbieten soll, so wird er daher nach Möglichkeiten suchen, das von ihm zu übernehmende Risiko seinerseits zumindest teilweise auf Dritte zu verlagern. In Betracht kommen hierfür neben den klassischen Gestaltungsmöglichkeiten der Mitversicherung und der Rückversicherung auch eine Risikoverlagerung auf den Staat sowie der Einsatz innovativer Kapitalmarktinstrumente.

\section{Mitversicherung}

Im Versicherungsgeschäft ist es heute üblich geworden, dass größere Risiken nicht von einem Versicherer allein getragen werden, sondern dass sich mehrere Versicherer in Gestalt der Mitversicherung zusammenfinden. Darunter versteht man die einvernehmliche Beteiligung mehrerer Versicherer an der Deckung eines bestimmten Interesses in der Weise, dass jeder Versicherer gegenüber dem Versicherungsnehmer eine bestimmte Quote (Teilschuld i.S. von $\S 420 \mathrm{BGB}$ ) übernimmt. ${ }^{26}$ Dies führt zu einer Art Sozialisierung des Risikos auf Seiten des Versicherers, gleichsam spiegelbildlich zur Sozialisierung auf Seiten der Versicherungsnehmer.

\section{Rückversicherung}

Der dem Versicherungsnehmer als Vertragspartner gegenübertretende Versicherer kann sich auch dadurch absichern, dass er für das gesamte Risiko oder einen Teil davon einen oder mehrere Rückversicherer einschaltet. Beim Rückversicherer handelt es sich gleichsam um den Versicherer des Versicherers; letzterer wird zur Abgrenzung auch als Erstversicherer bezeichnet. Dieses Modell bietet vielfach die Gewähr dafür, dass die dauerhafte Erfüllbarkeit der vom Erstversicherer abgeschlossenen Versicherungsverträge sichergestellt ist. Die Rückversicherung ist nach wie vor das wichtigste Instrument für einen (Erst-)Versicherer, um Katastrophenrisiken abzudecken. ${ }^{27}$

Allerdings gilt auch im Verhältnis von Erst- und Rückversicherer der Grundsatz der Vertragsfreiheit, und bei Katastrophenrisiken finden sich Rückversicherer häufig nicht zu einer Deckung bereit. ${ }^{28}$ Aus ihrer Sicht bestehen nämlich dieselben Kalkulationsschwierigkeiten wie für den Erstversicherer. Zudem hat sich der oben aufgezeigte sprunghafte Anstieg der Katastrophenschäden in den vergangenen zwei Jahrzehnten auch in einer deutlichen Erhöhung der Rückversicherungsprämien niedergeschlagen.

25 Sigma Nr. 1/2004, S. 14.

26 Näher Armbrüster, in: Beckmann/Matusche-Beckmann (Hrsg.), VersicherungsrechtsHandbuch, 2004, § 6 Rn. 40 ff.

27 Sigma Nr. 1/2004, S. 14.

28 Vgl. Nell, Staatshaftung für Terrorrisiken (Fn. 12), S. 2. 


\section{Risikoverlagerung auf den Staat}

Die Aufgaben des Staates im Bereich der Privatversicherung sind grundsätzlich auf den Schutz der Interessen der Versicherten beschränkt, wobei als ein »Subziel« der Schutz der Wirtschaftsfunktion Versicherung anzusehen ist. ${ }^{29}$ Das Instrument zur Verfolgung dieser Aufgaben ist die Aufsicht über die Geschäftstätigkeit der Versicherer, die durch die Bundesanstalt für Finanzdienstleistungsaufsicht wahrgenommen wird. Im Rahmen der Finanzaufsicht hat die Aufsichtsbehörde insbesondere auf die dauerhafte Erfüllbarkeit der Versicherungsverträge zu achten (s. dazu § 81 Abs. 1 S. 5 VAG). Im Übrigen tritt der Staat nach der schrittweisen Öffnung der europäischen Versicherungsmärkte ${ }^{30}$ am Markt nicht mehr als Monopolversicherer auf; vielmehr stehen die verbliebenen öffentlich-rechtlichen Versicherer heute in Konkurrenz zu privaten Anbietern.

Bisweilen nimmt der Staat jedoch auch noch in einer anderen Weise Einfluss auf das Marktgeschehen, nämlich indem er selbst anstelle eines Versicherers ein Risiko übernimmt. Dies ist etwa der Fall bei der sog. Staatsgarantie für den Ersatz von Schäden an Kunstwerken, die eine entsprechende privatversicherungsrechtliche Deckung entbehrlich machen soll. ${ }^{31}$ Ein Beispiel hierfür bietet die auf Bundesebene für die MoMA-Ausstellung 2004 in Berlin abgegebene Staatsgarantie. Bei derartigen Garantien geht es der öffentlichen Hand darum, die angesichts hoher Versicherungsprämien bisweilen gefährdete Durchführbarkeit von Ausstellungsprojekten zu erleichtern, mithin um den Gemeinwohlbelang der Kultur.

Bei Katastrophenrisiken kann ein anderes Interesse des Gemeinwohls, nämlich der Schutz vor existenzbedrohenden Schäden, dazu führen, dass der Staat sich im Privatversicherungsmarkt betätigt. Ein Beispiel hierfür, auf das zurückzukommen sein wird (unten VI 3 b), ist die Beteiligung des Staates an dem in Deutschland eingerichteten System des Deckungsschutzes für Terrorrisiken. Der Staat übernimmt hier ähnlich wie ein Rückversicherer im Umfang von 10 Mrd. Euro die Rückdeckung der bei der Extremus AG als Erstversicherer bestehenden Risiken. ${ }^{32}$ Jene Gesellschaft zahlt dafür ein laufendes Entgelt, mit dem die Bereitstellung des staatlichen Haftungsfonds abgegolten ist; wird der Staat nach Eintritt des Versicherungsfalls in Anspruch genommen, so muss der Erstversicherer die empfangene Summe - anders als nach manchen ausländischen Konzepten (s. dazu noch unten VI 3 b) - nicht teilweise wieder zurückzahlen.

\section{Risikoverlagerung auf den Kapitalmarkt (Alternativer Risikotransfer)}

In jüngster Zeit sind am Kapitalmarkt vor allem unter dem Eindruck verstärkt auftretender Naturkatastrophen sowie der zunehmenden Gefahr von Terroranschlägen neue Produkte entwickelt worden. Sie dienen dazu, über die vorhandenen Rück-

29 Kaulbach, in: Fahr/Kaulbach, VAG, 3. Aufl. 2003, Vor § 1 Rn. 5.

30 Zu dieser Entwicklung s. Kaulbach, (Fn. 29), Vor $\$ 1$ Rn. 16 ff.; Prölss/Armbrüster, DZWir 1993, 397, $398 \mathrm{ff}$.

31 Instruktiv hierzu Gärtner, NJW 1991, 2993, $3000 \mathrm{f}$.

32 S. dazu Gas/Thomann, ZVersWiss 2003, 697, 705. 
versicherungsmöglichkeiten (s. oben 2) hinaus zusätzliche Deckungskapazitäten zu schaffen. Für diese Finanzierungsinstrumente hat sich der Ausdruck Alternativer Risikotransfer (ART) eingebürgert; man spricht auch von Insurance-Linked Securities (ILS). Im Wesentlichen handelt es sich um die Verbriefung von Versicherungsrisiken dadurch, dass klassische Finanzmarktprodukte für den Versicherungsmarkt angepasst werden.

$\mathrm{Zu}$ nennen sind insbesondere Risikoanleihen (sog. Cat Bonds, Katastrophenanleihen). Vereinfacht gesagt, hängt die Verzinsung oder Rückzahlung solcher Anleihen vom Eintritt des vereinbarten Versicherungsfalls (Katastrophenereignis) ab. ${ }^{33} \mathrm{Im}$ Einzelnen haben sich eine Reihe von Gestaltungen herausgebildet. Sie unterscheiden sich im Ergebnis insbesondere dadurch, dass dem Risikoträger (Erst- und Rückversicherer oder auch Industrieunternehmen) ein verschiedenartiges Restrisiko verbleibt.

Die wichtigsten Modelle seien hier in der gebotenen Kürze skizziert. Anleihen auf Entschädigungsbasis bieten dem Risikoträger eine umfassenden Absicherung: Zugrunde gelegt wird der konkrete Versicherungsbestand, der mit dem Index übereinstimmt, an den die Transaktion gekoppelt ist. Bei sog. Branchenindextriggern ist hingegen nicht der konkrete Versicherungsbestand maßgeblich, sondern das Gesamtvolumen der durch die Katastrophe ausgelösten Branchenschäden. $\mathrm{Zu}$ leisten ist ein bestimmter Prozentsatz dieser Gesamtsumme, wobei Unter- und Obergrenzen vorgesehen werden. Noch abstrakter wird die Auszahlung bei sog. Modellschadentriggern bestimmt; hier sind nicht die tatsächlich im Bestand des Risikoträgers eingetretenen Schäden maßgeblich, sondern die aufgrund eines physikalischen Schadensberechnungsmodells zu erwartenden Schäden. Rein parametrische oder physikalische Trigger führen zu einem Zahlungsanspruch, der allein von Ort und Schwere der Katastrophe abhängt. Dieses Modell lässt sich durch parametrische Indizes verfeinern, die eine von der örtlichen Risikoexponierung des Risikoträgers abhängige Gewichtung vornehmen und damit wieder auf den konkreten Risikobestand abstellen. Jene Gestaltung ist in der Praxis am meisten verbreitet. ${ }^{34}$

Das Marktvolumen dieses Sektors ist in den letzten Jahren sprunghaft gestiegen. ${ }^{35}$ Cat Bonds ermöglichen dem Risikoträger eine Absicherung gegen Katastrophenrisiken auf mehrere Jahre, ohne dass ein Gegenpartei- oder Kreditrisiko besteht. Dabei können mehrere Risiken in einem Cat Bond zusammengefasst werden; in letzter Zeit werden jedoch vermehrt auf einzelne Katastrophenrisiken bezogene Anleihen emittiert. Sie lauten etwa auf »Erdbeben Japan«, »Sturm Europa« oder »Sturm USA«.

Hinzu kommen Versicherungsderivate wie Futures, Swaps, Forwards und Optionen, denen ein versicherungstechnischer Index zugrunde liegt. Gewählt werden in der Regel spezielle Schadensindices, in denen die Entwicklung abstrakt definierter Großund Katastrophenschäden wiedergeben wird. Solche Schadensindices werden von der US-amerikanischen Einrichtung Property Claims Service (PCS) erstellt, einer Abteilung des Insurance Services Office, Inc. (ISO). Letztere ist eine Organisation der US-

33 K. Weber, ZfV 2005, 357, 359 ff.

34 Sigma Nr. 1/2004, S. 16.

35 Sigma Nr. 1/2004, S. 14. 
amerikanischen Versicherungswirtschaft, die sich mit der Erfassung und Schätzung von Katastrophenschäden befasst.

Schließlich ist auch Bedingtes Kapital (Contingent Capital) zu nennen. Damit wird es dem Versicherer ermöglicht, gegen eine festgelegte Optionsprämie bei Eintritt des Versicherungsfalls (Katastrophe) Eigen- oder Fremdkapitaltitel, etwa Vorzugsaktien oder Genussrechte, an Kapitalanleger zu veräußern. Dadurch wird dem Umstand Rechnung getragen, dass eine solche Kapitalbeschaffung nach Eintritt eines Großschadens anderenfalls häufig sehr schwierig wäre. Von der Rückversicherung unterscheidet sich dieses Kapitalmarktinstrument dadurch, dass die versicherungstechnischen Risiken beim Versicherer verbleiben und ihm lediglich die Kapitalbeschaffung nach Realisierung der versicherten Gefahr erleichtert wird.

Aus Sicht der Versicherer (Erst- oder Rückversicherer) erschließt sich über diese innovativen Kapitalmarktprodukte eine neue Möglichkeit der Risikodeckung. Damit lassen sich insbesondere Kapazitätsengpässe in der Rückversicherung überwinden, etwa hinsichtlich Hurricane- oder Sturmrisiken. Hinzu kommt die Möglichkeit, vorübergehende Liquiditätsengpässe zu bewältigen, wie sie etwa aufgrund der Börsenrückgänge seit dem Jahr 2000 aufgetreten sind. Gegenüber der Rückversicherung bringen die innovativen Kapitalmarktinstrumente freilich auch Nachteile mit sich. So entfällt etwa die durch einen Rückversicherer üblicherweise gebotene Unterstützung bei der Prämientarifierung. ${ }^{36}$ Auch kann es dazu kommen, dass die Anleger die von ihnen eingegangenen Verpflichtungen nicht erfüllen können. ${ }^{37}$

Für die Kapitalanleger, die derartige Produkte erwerben, liegen die Vorzüge vor allem in erhöhten Renditen. Hinzu kommt die Möglichkeit, die Anlagerisiken stärker zu diversifizieren, da die innovativen Instrumente in gewissem Umfang von der Entwicklung der übrigen Kapitalmärkte abgekoppelt sind.

\section{Umfang des Versicherungsschutzes für die einzelnen Katastrophenrisiken}

\section{Naturkatastrophen}

a) Erdbeben (einschließlich Seebeben, Tsunamis)

$\mathrm{Zu}$ den Naturkatastrophen zählen Erdbeben (einschließlich Seebeben, Tsunamis). ${ }^{38}$ Das Erdbebenrisiko ist neben dem Kriegsrisiko (dazu s. unten $3 a)^{39}$ in der Feuerversicherung nach dem bereits erörterten $\S 84$ VVG Gegenstand eines gesetzlichen Risikoausschlusses. Außerhalb der Feuerversicherung wird dieser Ausschluss regelmäßig in den AVB vereinbart. ${ }^{39}$

36 Darauf verweist auch K. Weber, ZfV 2005, 357, 363.

37 Hiervor warnt Wolgast, ifo-Schnelldienst 24/2001, S. 12, 13.

38 Eine Übersicht über die bedeutendsten Erdbeben und Tsunamis zwischen 1900 und 2004 findet sich unter http://www.munichre.com/assets/pdf/press/pr/2004_12_28_press_release_200_ app_02_de.pdf.

39 Vgl. nur \$ 9 Nr. 1 a VGB 88 (abrufbar unter http://www.gdv.de/Service/inhaltsseite590.html). 
Tsunamis, die seit der Katastrophe im Pazifikraum Ende 2004 verstärkt in den Blick gerückt sind, können durch verschiedene geologische Ereignisse ausgelöst werden. Neben Erdbeben auf dem Meeresgrund (Seebeben) sind als mögliche Ursachen auch Vulkanausbrüche oder Erdrutsche unter der Meeresoberfläche, durch ins Wasser stürzende Erd- oder Felsmassen sowie Meteoriteneinschläge zu nennen. ${ }^{40}$ Als Folgeerscheinung von Erdbeben sind Tsunamis versicherbar. ${ }^{41}$

b) Überschwemmung

Überschwemmungsschäden sind in manchen Versicherungszweigen eingeschlossen, so etwa in der Kfz-Kaskoversicherung. In den Allgemeinen Gebäudeversicherungsbedingungen sind sie hingegen häufig ausgeschlossen. ${ }^{42}$ Dieser Ausschluss kann durch die Vereinbarung Besonderer Bedingungen für die Versicherung weiterer Elementarschäden abbedungen werden. ${ }^{43}$ Auch soweit eine solche Zusatzversicherung besteht, enthält diese keinen umfassenden Versicherungsschutz gegen Überschwemmungsschäden. So können etwa Schäden durch Sturmflut vom Versicherungsschutz ausgenommen sein. ${ }^{44}$ Definiert wird der Begriff der Überschwemmung in den Bedingungen zumeist als eine Überflutung des Grund und Bodens des Versicherungsortes durch Ausuferung von oberirdischen Gewässern oder Witterungsniederschlägen. ${ }^{45}$

Die aus der Zeit der Staatlichen Versicherung der DDR stammenden Altverträge sahen in $\S 1$ Abs. 1 a der Allgemeinen Bedingungen für die freiwillige Versicherung von Gebäuden freilich auch für Gebäude eine Deckung von Überschwemmungsschäden vor. ${ }^{46}$ Soweit solche Policen nach der deutschen Wiedervereinigung nicht gekündigt wurden, boten sie mithin Schutz vor den Folgen des Elbehochwassers von 2002. Dieses »Jahrhunderthochwasser « hat in Deutschland den Blick verstärkt auf den Schutz vor Überschwemmungsschäden gelenkt, der zuvor verbreitet als ein regional eng begrenztes Problem von küstennahen Orten und bestimmten Flusslagen aufgefasst worden war.

Überschwemmungen treten weltweit auf, und zwar mit zunehmender Tendenz. ${ }^{47}$ Als Ursachen lassen sich neben der globalen Klimaänderung insbesondere die Besiedelung und Industrialisierung gefährdeter Gebiete, bestimmte Entwicklungen beim

40 Sigma Nr. $1 / 2005$, S. 8.

41 Sigma Nr. 1/2005, S. 12.

42 Vgl. § 9 Nr. 4 b VGB 88 (abrufbar unter http://www.gdv.de/Service/inhaltsseite590.html).

43 Vgl. BGH VersR 2005, 828 (betr. Wohngebäudeversicherung).

44 Vgl. § 3 Nr. 2 b Besondere Bedingungen für die Versicherung weiterer Elementarschäden bei gewerblichen Risiken (BEG 2000) (abrufbar unter http://www.gdv.de/Service/ inhaltsseite 590.html).

45 Vgl. BGH VersR 2005, 828; OLG Karlsruhe NVersZ 2001, 570.

46 Abgedruckt in: Martin-Luther-Universität Halle-Wittenberg und Hauptverwaltung der Staatlichen Versicherung der Deutschen Demokratischen Republik (Hrsg.), Versicherungsrecht. Textausgabe der Rechtsnormen und Vertragsbedingungen für die Sach-, Haftpflichtund Personenversicherungen in der Deutschen Demokratischen Republik mit Anmerkungen und Sachregister, Berlin 1982, S. 67.

47 Münchener Rückversicherungs-Gesellschaft (Hrsg.), Überschwem mung und Versicherung, 1997, S. 37. 
Flussbau, eine fortschreitende Versiegelung der Erdoberfläche und Eingriffe in die Vegetation ausmachen. Eine entsprechende Analyse der lokalen Gegebenheiten kann neben der statistischen Entwicklung wichtige Anhaltspunkte für die Kalkulation des Risikos bieten. In Deutschland sind die Bundesländer aufgrund der jüngeren Gesetzgebung zum Hochwasserschutz verpflichtet, Karten von Überschwemmungsgebieten und überschwemmungsgefährdeten Gebieten zu erstellen; auch aus solchem Material können die Versicherer zusätzlich zu ihren eigenen Ermittlungen kalkulationsrelevante Informationen entnehmen.

Tritt eine Überschwemmungskatastrophe ein, so wird nicht selten in der Politik der Ruf nach der Einführung einer gesetzlichen Pflichtversicherung von Überschwemmungsschäden laut, entweder flächendeckend oder zumindest für besonders gefährdete Gebiete. ${ }^{48}$ Gesetzgeberische Maßnahmen ${ }^{49}$ haben sich daran bisher freilich regelmäßig nicht angeschlossen. Dafür lassen sich mehrere Gründe feststellen: Zunächst dient die klassische Pflichtversicherung dazu, die Interessen Dritter (insbesondere: der durch einen Verkehrsunfall Geschädigten bei der Kfz-Haftpflichtversicherung) und nicht diejenigen des Versicherungsnehmers selbst zu schützen. Verfassungsrechtlich wäre ein staatlicher Zwang zur Eigenvorsorge sub specie der grundgesetzlich gewährleisteten Privatautonomie keineswegs unbedenklich. Hinzu kommt, dass der zwingende Einschluss von Überschwemmungsschäden manchen Versicherern, die über einen hohen Sachversicherungsbestand in besonders gefährdeten Gebieten verfügen, Kapazitätsprobleme bereiten dürfte, ${ }^{50}$ denen er bei einer Pflichtversicherung auch nicht ohne weiteres durch eine Kündigung entgegnen könnte. Der Versicherungsnehmer wiederum müsste im Interesse einer bezahlbaren Prämie erhebliche Selbstbehalte in Kauf nehmen. Schließlich zeigt der Staat (Bund und Länder) anders als hinsichtlich des Terrorrisikos bislang keine Bereitschaft, sich an der Deckung von Überschwemmungsschäden durch eine Staatsgarantie zu beteiligen.

c) Sturm, Hagel

Im Gegensatz zum Überschwemmungsrisiko kann das Risiko von Sturmschäden vergleichsweise leicht am Markt versichert werden. Dieses Risiko lässt sich durch einen selbstständigen Versicherungsvertrag decken oder - und dies ist gerade im privaten Bereich häufig - in Gestalt einer kombinierten Deckung im Rahmen der Hausrat- oder der Wohngebäudeversicherung. ${ }^{51}$ Sturm wird dabei gemeinhin als eine wetterbedingte Luftbewegung von mindestens Windstärke 8 definiert $^{52}$. Anders als dies hinsichtlich

48 Vgl. etwa die Presserklärung des Bayerischen Ministerpräsidenten zum Hochwasser Pfingsten 1999, S. 9 (abrufbar unter http://www.bayern.de/Presse-Info/Regierungserklaerungen/ pdf/reg_990608.pdf); Schwarze/Wagner, Hochwasserkatastrophen in Deutschland: Über Soforthilfe hinausdenken, Wochenbericht des DIW Berlin 35/02 (abrufbar unter: http:// www.diw.de/deutsch/produkte/publikationen/wochenberichte/docs/02-35-2.html ).

49 Für kombinierte Lösungen von Versicherung und staatlichen Beihilfen bereits Scheying, JZ 1976, $635 \mathrm{ff}$.

50 S. dazu etwa den Bericht in ZfV 2005, 349.

51 Martin, Sachversicherungsrecht, 3. Aufl. 1992, E II Rn. 1.

52 Näher Wussow, VersR 2000, 679, 680. 
sonstiger vom Deckungsschutz erfasster Schadensursachen der Fall ist, sind nach den Bedingungen regelmäßig nicht sämtliche adäquaten Folgen eines Sturms versichert, sondern nur bestimmte, näher beschriebene Kausalverläufe. Kombiniert wird die Deckung von Sturmschäden zumeist mit einer Versicherung gegen Hagelschäden. Dadurch wird die Gefahr von Rechtsunsicherheiten vermieden, da es sich oftmals um gleichzeitig eintretende und in ihren Wirkungen nur schwer voneinander abgrenzbare Risiken handelt ${ }^{53}$.

d) Dürre, Flächenbrand

Das Risiko, durch Dürre (längere Trockenperioden) oder durch Flächenbrände eine Vermögenseinbuße zu erleiden, besteht vornehmlich in der Landwirtschaft. Sofern es um Waldbrände geht, gibt es eine spezielle Waldbrandversicherung. Dabei leistet der Versicherer regelmäßig nicht nur Ersatz für die zerstörten Wald- und Holzbestände, sondern darüber hinaus auch für Schäden, die durch Löscharbeiten, auch Gegenfeuer, Niederreißen oder notwendiges Ausgraben entstehen. ${ }^{54}$ Eine vergleichbare Versicherung für Ernteausfälle infolge anhaltender Dürreperioden findet sich auf dem deutschen Versicherungsmarkt - anders als etwa in manchen anderen europäischen Staaten oder in den Vereinigten Staaten - nur vereinzelt. Ein Grund dafür, dass dieser Deckungsschutz bisher in Deutschland kaum Verbreitung gefunden hat, ${ }^{55}$ dürfte neben dem vergleichsweise eher geringen Schadensrisiko in der Tatsache liegen, dass die Versicherungsprämien hierzulande abweichend von anderen Ländern nicht staatlich subventioniert werden. ${ }^{56}$

e) Kälte, Frost

Kälte- und Frostschäden spielen vor allem bei der Versicherung von Wohngebäuden eine Rolle. Betroffen sind hier insbesondere Wasserleitungen und Heizungsanlagen. Soweit sich aus einem durch Frost verursachten Wasserleitungsschaden weitere Vermögensschäden ergeben, können diese im gewerblichen Bereich durch die Vereinbarung Besonderer Bedingungen zur Feuer-Betriebsunterbrechungs-Versicherung gedeckt werden. Im landwirtschaftlichen Bereich, etwa für Winzer, werden insbesondere kombinierte Hagel- und Frostversicherungspolicen angeboten.

f) Sonstiges (insbesondere: Lawinen)

Auch eine Reihe sonstiger Risiken können Folgen mit katastrophalen Ausmaßen nach sich ziehen. Zu nennen ist etwa das Lawinenrisiko. So wurde unter dem Eindruck des Lawinenunglücks in Galtür Anfang 1999 eine entsprechende Deckung als Annex zur

53 Ausführlich dazu Martin, Sachversicherungsrecht (Fn. 51), E II Rn. 6 ff.

54 Vgl. § 1 Allgemeine Versicherungs-Bedingungen für Waldbrandversicherung (abrufbar unter http://www.gdv.de/Service/inhaltsseite590.html).

55 Zum (Versuchs-)Modell einer Ernteausfallversicherung s. http://www.ernteausfallversicherung.de/.

56 Vgl. Wilkens, Versicherung gegen Katastrophen 2003 (abrufbar unter http://www.landvolk.net/2838.htm). 
Feuerversicherung auf den österreichischen Markt gebracht, wenn auch regelmäßig mit vergleichsweise niedrigen Deckungssummen. ${ }^{57}$

Gerade Schadensfälle durch Lawinen verdeutlichen zugleich die Berührungspunkte, die zwischen Naturkatastrophen und sog. Man-made-Katastrophen bestehen. ${ }^{58}$ So kann eine Lawine durch Menschen ausgelöst werden oder von menschlichem Verhalten unabhängige Ursachen haben. Bezieht man zudem noch Aspekte wie die Abholzung von Berghängen und die fortschreitende Bebauung in die Betrachtung mit ein, so verschwimmen die Grenzen noch mehr.

\section{Katastrophen aufgrund menschlichen Versagens}

a) Großbrände, Explosionen, Kernenergie

Großbrände und Explosionen sind gerade im Bereich der Industrieversicherung ein Hauptgrund für den Abschluss einer Feuerversicherung. Besondere Gefahren bestehen hier etwa bei chemischen Anlagen, Öltanks oder Gasspeichern. Herausgehobene Bedeutung hat die Kernenergie. Das Risiko der friedlichen Nutzung der Kernenergie wird in Deutschland seit 1955 in den Allgemeinen Feuerversicherungsbedingungen regelmäßig ausgeschlossen. ${ }^{59}$ Der Ausschluss bezieht sich dabei auf Schäden, die durch den Betrieb von Kernkraftwerken entstehen können, nämlich den Ausbruch von Bränden, die durch Kernspaltungsprozesse ausgelöst werden, sowie durch solche Prozesse verursachte Strahlungs- und Kontaminationsschäden. ${ }^{60}$ Die Dimensionen eines möglichen Schadenszenarios sind durch die Reaktorkatastrophe von Tschernobyl im Jahr 1986 deutlich geworden, deren Folgen auch in Westeuropa gravierend waren. Den Ersatz derartiger Schäden regelt in Deutschland das AtomG, wonach den Betreiber einer Kernenergieanlage eine Gefährdungshaftung trifft. Das Risiko, aufgrund des AtomG in Anspruch genommen zu werden, decken die Betreiber zumindest zum Teil über eine Haftpflichtversicherung ab. Zu diesem Zweck besteht die Deutsche KernreaktorVersicherungsgemeinschaft (DKVG), die in Form eines Pools Haftpflicht- und Sachversicherungsschutz in Bezug auf die mit der Errichtung und dem Betrieb von Kernreaktoren und ähnlichen Anlagen verbundenen Risiken bietet. Die DKVG arbeitet überwiegend als Rückversicherungspool im Austausch mit ausländischen Atompools.

b) Verkehrskatastrophen (Luft- und Raumfahrt, Schifffahrt, Bahn, Kfz)

Anders als in vielen anderen Bereichen besteht für die Deckung der Folgen von Verkehrskatastrophen regelmäßig die gesetzliche Pflicht zum Abschluss einer Versicherung. ${ }^{61}$ Dabei umfasst etwa die Luftfahrtversicherung das Risiko einer Beschädigung und des Verlusts am Boden, in der Luft, im Wasser und während eines Transportes; zugleich sind Lufthaftpflicht und Luftinsassenunfall gedeckt. Ähnlich wie bei der Ab-

57 Vgl. http://www.die-wirtschaft.at/ireds-711.html.

58 Weitere Beispiele bei von Arnauld, AVR 43 (2005), 279, $281 \mathrm{f}$.

59 Vgl. § 1 Nr. 7 AFB 87 (abrufbar unter http://www.gdv.de/Service/inhaltsseite590.html).

60 Johannsen/Johannsen, in: Bruck/Möller/Sieg/Johannsen (Fn. 17), Anm. H 39.

61 Vgl. etwa § 43 LuftVG. 
sicherung des Kernenergierisikos ist auch hier ein Pool (sog. Luftpool) geschaffen worden, dem sich die meisten deutschen Transportversicherer angeschlossen haben. Im Bereich der Bahn besteht zur Absicherung der Risiken der Versicherungsverband Deutscher Eisenbahnen. In diesem Verband sichern die zumeist öffentlich-rechtlichen Betreiber ihr Sach- und Haftpflichtrisiko über ein Umlageverfahren ab. ${ }^{62}$

\section{c) Sonstiges}

Neben den genannten Katastrophen sind etwa noch Gruben- und Minenunglücke oder Gebäudeeinstürze zu nennen. Letztere können - je nach Ursache - in der Feuer- oder Wohngebäudeversicherung gedeckt sein.

\section{Vorsätzlich herbeigeführte Katastrophen}

a) Krieg und innere Unruhen

Wie bereits im Zusammenhang mit den gesetzlichen Vorgaben (oben II 4) ausgeführt, sieht § 84 VVG einen - freilich abdingbaren - Ausschluss des Kriegsrisikos in der Feuerversicherung vor. Erfasst werden davon nach dem Gesetzeswortlaut Schäden, die »durch Maßregeln verursacht [werden], die im Kriege oder nach Erklärung des Kriegszustandes von einem militärischen Befehlshaber angeordnet worden sind ${ }^{63}$ Darüber hinausgehend wird das Kriegsrisiko in Allgemeinen Versicherungsbedingungen verbreitet in weiterem Umfang ausgeschlossen. So heißt es etwa in den Musterbedingungen zur Feuerversicherung: »Die Versicherung erstreckt sich ohne Rücksicht auf mitwirkende Ursachen außerdem nicht auf Schäden an versicherten Sachen und nicht auf versicherte Kosten, die durch Kriegsereignisse jeder Art, innere Unruhen, Erdbeben oder Kernenergie verursacht werden. ${ }^{64}{ }^{6}$ Vergleichbare Klauseln enthalten etwa auch die Musterbedingungen zur Verbundenen Gebäudeversicherung $^{65}$, zur Hausratversicherung ${ }^{66}$ oder zur Maschinenversicherung ${ }^{67}$.

Hintergrund dieser sog. Kriegsklauseln ist der Umstand, dass es sich um ein Risiko handelt, das hinsichtlich der Wahrscheinlichkeit seiner Verwirklichung und des Schadensumfangs als kaum kalkulierbar erscheint. ${ }^{68}$ Versicherungsrechtlich wird Krieg dabei nicht im völkerrechtlichen Sinne verstanden, sondern umfasst über die im Völkerrecht für Beginn und Ende maßgeblichen Zäsuren (förmliche Kriegserklärung oder Feststellung des Verteidigungsfalls; Waffenstillstand oder Friedensvertrag) hinaus jeglichen tatsächlichen kriegerischen Gewaltzustand. ${ }^{69}$

62 S. http://www.vvde.de/main/index $2 . h t m l$.

63 Einen gleichlautenden Ausschluss enthält $§ 117$ Nr. 2 VVG für die Tierversicherung.

$64 \S 1$ Nr. 7 AFB 87 (abrufbar unter http://www.gdv.de/Service/inhaltsseite590.html).

$65 \S 9$ Nr. 1 b VGB 88 (abrufbar unter http://www.gdv.de/Service/inhaltsseite590.html).

$66 \S 9$ Nr. 1 b VHB 92 (abrufbar unter http://www.gdv.de/Service/inhaltsseite590.html).

$67 \S 2$ Nr. 5 a AMB 91/97 (abrufbar unter http://www.gdv.de/Service/inhaltsseite590.html).

68 Dahlke, VersR 2003, 25, 27; Fricke, VersR 1991, 1098, 1100; ders., VersR 2002, 6, 7; Glotzmann, VersR 1975, 784, 785; s. auch (im Kontext von § 84 VVG) oben bei Fn. 17.

69 Weitere Einzelheiten bei Dahlke, VersR 2003, 25, 28; Fricke, VersR 1991, 1098, 1099; für ein Verständnis im völkerrechtlichen Sinne Langheid, in: Römer/Langheid, VVG, 2003, § 84 Rn. 3. 
Regelmäßig wird der Ausschluss auch auf andere sog. politische Risiken ausgedehnt, namentlich auf innere Unruhen. ${ }^{70}$ Letztere unterscheiden sich vom Krieg dadurch, dass sie sich innerhalb des Territoriums eines einzigen Staates abspielen. Nach einer bereits im Jahre 1923 vom Reichsgericht ${ }^{71}$ begründeten und vom Bundesgerichtshof ${ }^{72}$ aufgegriffenen Rechtsprechung sind innere Unruhen dann anzunehmen, wenn »zahlenmäßig nicht unerhebliche Teile des Volkes in einer die öffentliche Ruhe und Ordnung störenden Weise in Bewegung geraten und Gewalttätigkeiten gegen Personen oder Sachen verüben « ${ }^{73}$

Bisweilen wird davon ausgegangen, dass die genannten politischen Risiken per se unversicherbar seien, da sie sich einer statistischen Risikoerhebung entzögen. ${ }^{74}$ Indessen bedarf es insoweit einer Differenzierung. Politische Risiken mögen aus Sicht eines Risikoträgers faktisch unversicherbar sein, sofern Versicherungsschutz am Markt nicht angeboten wird; ${ }^{75}$ aus Rechtsgründen unversicherbar sind sie jedoch nicht.

\section{b) Terroranschläge}

aa) Terrorrisiken und Allgemeine Versicherungsbedingungen

Der Deckungsschutz für Terrorrisiken ist nach den Anschlägen vom 11.9.2001 ins Zentrum der Aufmerksamkeit gerückt. ${ }^{76}$ Bei derartigen Risiken sind die Kalkulationsschwierigkeiten besonders groß, da es keinerlei verlässliche Schadensprognosen gibt $^{77}$ und zugleich die Höhe möglicher Schäden als praktisch unbegrenzt erscheint.

Eine allgemein anerkannte versicherungsspezifische Definition des Begriffs »Terror « fehlt bislang. Insbesondere hat der Gesetzgeber keine Legaldefinition ins Versicherungsvertragsgesetz aufgenommen; vielmehr befasst sich dieses Gesetz überhaupt nicht eigens mit Terrorschäden. Auch in den Allgemeinen Versicherungsbedingungen findet sich der Ausdruck »Terror « bislang nur vereinzelt als Gegenstand von Risikoausschlüssen (zur Definition der Extremus-Terrorversicherung s. unten bb). ${ }^{78}$ So besteht in der Seegüterversicherung ein Ausschluss für Schäden aufgrund von »terroristischen oder politischen Gewalthandlungen $\ll .{ }^{79}$ Angesichts der geringen Bedeutung der Kategorie »Terror « in den Versicherungsbedingungen hatte sich auch die versicherungsrechtliche Judikatur bislang nicht näher mit der Auslegung des Begriffs zu befassen. ${ }^{80}$

70 S. etwa $§ 1$ Nr. 7 AFB 87. Mitversichert sind innere Unruhen hingegen nach den Musterbedingungen zur Transportversicherung (Nr. 2.4.1.1. der DTV-Güter 2000/2004, abrufbar unter http://www.tis-gdv.de/tis/bedingungen/avb/ware/w_0405_01_volle_deckung.pdf).

71 RGZ 108, 188, 190.

72 BGHZ 6, 28, 32 = VersR 1952, 177; BGH VersR 1975, 126.

73 Einzelheiten hierzu bei Dimski, VersR 1999, 804, 807 f.; U. Hübner, ZVersWiss 1981, 1, 37.

74 Dimski, VersR 1999, 804, 807; Horster, Der Ersatz von Tumultschäden durch Staat und Versicherung, 1988, 807.

$75 \mathrm{Zu}$ diesem Begriffsverständnis s. Wolgast, ifo-Schnelldienst 24/2001, S. 12.

76 Hübner, in: Festschrift für Kollhosser, 2004, Bd. I, S. 179 ff.; Langheid/Rupietta, NJW 2005, 3233 ff.; Wolgast, ifo-Schnelldienst 24/2001, S. 12, 14.

77 Wolgast, ifo-Schnelldienst 24/2001, S. 12.

78 Dahlke, VersR 2003, 25, 26; Hübner (Fn. 76), S. 179, 183.

79 Nr. 1.1.2.2 der Allgemeinen deutschen Seeversicherungs bedingungen/Besondere Bestimmungen für die Güterversicherung (ADS 73/84).

80 Dahlke, VersR 2003, 25, 26. 
Insoweit fällt der Unterschied zu Ausschlussklauseln für Kriegsrisiken auf, die in den Bedingungswerken verbreitet anzutreffen sind (s. oben a). Offenkundig war das Terrorrisiko aus Sicht der Verfasser von AVB bislang als möglicher Gegenstand eines Risikoausschlusses zu vernachlässigen. ${ }^{81}$ Dies ist rückblickend nachvollziehbar, da zumindest in Deutschland die Versicherungswirtschaft in der Vergangenheit nicht in nennenswertem Umfang mit der Regulierung von Terrorschäden befasst war. So zielte insbesondere der politisch motivierte Terror der RAF nicht darauf ab, möglichst hohe Personen- und Vermögensschäden zu verursachen, sondern er bezweckte in erster Linie, einzelne symbolträchtige Repräsentanten von Staat und Wirtschaft zu treffen. Mit dem offiziellen Ende der RAF schien selbst diese - unter dem Blickwinkel der versicherten Risiken ohnehin begrenzte - Gefahr gebannt.

Diese Wahrnehmung von Terror als einem für die Versicherungswirtschaft eher zu vernachlässigenden Schadensrisiko hat sich mit der Häufung von Terroranschlägen in der westlichen Welt und zunehmend auch in Europa in der jüngsten Vergangenheit geändert. Die Terroranschläge vom 11.9.2001 haben allein in der Sach- und Betriebsunterbrechungsversicherung einen versicherten Gesamtschaden von über $20 \mathrm{Mrd}$. USD verursacht. $^{82}$

Aus heutiger Sicht stellt sich damit insbesondere die Frage, ob es einen sachlichen Grund dafür gibt, Terrorrisiken anders zu behandeln als die Risiken von Krieg und inneren Unruhen. In individuell ausgehandelten Verträgen über sog. Großrisiken (zum Begriff s. Art. 10 Abs. 1 EGVVG) wird eine Terrordeckung dem Vernehmen nach jedenfalls in Deutschland bereits heute verbreitet ausgeschlossen. Darin spiegelt sich das Interesse der Versicherungswirtschaft daran, die Kalkulationsschwierigkeiten und die Gefährdung der eigenen Leistungsfähigkeit zu vermeiden, die mit einer Deckung des Terrorrisikos verbunden sein können. Zugleich zeigt sich daran, dass die Versicherungsnehmer vielfach nicht bereit sind, eine Mehrprämie für den Einschluss des Terrorrisikos zu entrichten. Sehr deutlich kommt dies auch darin zum Ausdruck, dass das entsprechende Deckungsangebot der Extremus AG (s. dazu noch unten bb) am Markt bislang eher zurückhaltend aufgenommen wird. ${ }^{83}$

Jenseits der individuell ausgehandelten Versicherung von Großrisiken bleibt jedoch zu untersuchen, wie die Standardbedingungen mit Terrorrisiken umgehen. Für die Seeversicherung ist - wie dargelegt - in den Musterbedingungen bereits eine Gleichstellung terroristischer Gewalthandlungen mit Krieg vorgesehen. Dasselbe wird man in der Transportversicherung anzunehmen haben, auch wenn dort (allein) von politischen und nicht von terroristischen Gewalthandlungen die Rede ist. ${ }^{84}$ Auch lässt sich der vereinzelt anzutreffende Ausschluss bei höherer Gewalt ${ }^{85}$ durchaus auf Terrorrisiken beziehen. Für

81 S. aber bereits früh Glotzmann, VersR 1975, 784, $785 \mathrm{ff}$.

82 Sigma Nr. 1/2005, S. 34. Hinzu kommen noch Aufwendungen insbesondere in der Lebensund u.U. auch in der Haftpflichtversicherung.

83 S. dazu den Bericht in ZfV 2005, 267 (o. Verf.)

84 Dahlke, VersR 2003, 25, 30.

85 S. z.B. $§ 2$ Nr. 2 f AVBR 92/94 (Musterbedingungen für die Reisegepäckversicherung) oder Nr. 3.3.2 AVB Wassersportfahrzeuge 1985, Fassung 2004 (jeweils abrufbar unter http://www.gdv.de/Service/inhaltsseite590.html). 
die große Mehrzahl der Versicherungsbedingungen, in denen eine Gleichbehandlung nicht ausdrücklich vorgesehen oder ohne weiteres durch Auslegung anderer Ausschlusstatbestände als Krieg zu ermitteln ist, bleibt zu klären, ob ein Ausschluss von Terrorrisiken bereits jetzt anzunehmen ist oder ob er unter dem Gesichtspunkt systematischer Stimmigkeit zumindest in künftigen Bedingungswerken eingeführt werden sollte.

Parallelen zwischen Terror und Krieg bestehen bereits insofern, als es in allen Fällen um absichtliche physische Gewaltanwendung gegenüber Personen oder Sachen geht. ${ }^{86}$ Zudem ist jeweils der Schadenseintritt besonders schwer vorherzubestimmen und es drohen exorbitant hohe Schäden. ${ }^{87}$ Angesichts dieser Parallelen ist die Sichtweise vorgeschlagen worden, den versicherungsrechtlichen Kriegsbegriff um Terrorakte zu erweitern. ${ }^{88}$ Ausgenommen sollen nur einmalige Ereignisse mit lokal begrenzter Wirkung und untergeordneter Bedeutung sein. Diese Ansicht hat zweifelsohne den Vorteil, dass sie Abgrenzungsprobleme zwischen Terror und Krieg vermeidet. Zudem erübrigt es sich dann, auf konkrete Zurechnungsvoraussetzungen zu einer kriegführenden Partei abzustellen.

Eine andere Ansicht ${ }^{89}$ geht in der Gleichsetzung von Terror- mit Kriegsrisiken weniger weit. Entscheidend soll demnach sein, ob der Terrorakt in einem ursächlichen $\mathrm{Zu}$ sammenhang mit Kriegsereignissen steht. Dies könne auch dann der Fall sein, wenn der Anschlag außerhalb des Hoheitsgebiets der kriegführenden Parteien verübt werde. Einzelne Terrorakte mit nur begrenzter Wirkung stellten demgegenüber kein Risiko dar, das nach dem Sinn und Zweck des Kriegsrisikoausschlusses von diesem mit umfasst sein solle. Noch enger ist die Sicht, wonach es über den ursächlichen Zusammenhang hinaus der Zurechnung zu einer kriegführenden Partei bedarf: Demnach ist Terror dem Krieg dann versicherungsrechtlich gleichzustellen, wenn es um ein kriegsbedingtes Schadensereignis geht, das einer kriegführenden Partei zuzurechnen ist. ${ }^{90}$

Den genannten Ansichten lässt sich nicht immer klar entnehmen, ob der Ausschluss bestimmter Terrorrisiken sich bereits durch die Auslegung des Wortes »Krieg« ergeben soll oder aber im Wege einer ergänzenden Auslegung der Kriegsklausel. Ersteres kann nach der hier vertretenen Ansicht nur dazu führen, dass der Ausschluss solche Terrorakte erfasst, die einer kriegführenden Partei zuzurechnen sind. Eine ergänzende Vertragsauslegung könnte darüber hinaus zu einem umfassenderen Ausschluss führen. Sie setzt zunächst voraus, dass eine unbewusste Regelungslücke vorliegt. Davon kann hier jedenfalls für den Regelfall ausgegangen werden, da den Beteiligten das Terrorrisiko bei Vertragsschluss regelmäßig nicht vor Augen gewesen sein dürfte. ${ }^{91}$ Sodann müssten die Gemeinsamkeiten zwischen beiden Risiken eine Gleichbehandlung rechtfertigen, so dass die Parteien, wäre diese Frage zur Sprache gekommen, auch das Terrorrisiko ausgeschlossen hätten. Dies lässt sich für die vor dem 11.9.2001 abgeschlos-

86 Dahlke, VersR 2003, 25, 26.

87 Dahlke, VersR 2003, 25, 26.

88 Fricke, VersR 2002, 6, 10.

89 Grimm, Unfallversicherung, 3. Aufl. 2000, § 2 AUB Rn. 37.

90 Dahlke, VersR 2003, 25, 29; Hübner (Fn. 76), S. 179, 185 f.

91 Vgl. dazu bereits Fricke, VersR 1991, 1098, 1100. 
senen Verträge im Hinblick darauf nur schwer beurteilen, dass beide Parteien das Risiko seinerzeit als vernachlässigbar eingestuft hätten. Jedenfalls kann daraus, sofern nicht im Einzelfall weitere Umstände hinzukommen, nicht mit der für eine ergänzende Vertragsauslegung erforderlichen Klarheit der Schluss gezogen werden, dass der diesbezüglich beweisbelastete Versicherer auf einem Ausschluss bestanden hätte. Letzteres wird man für die Zeit seit dem 11.9.2001 zwar eher unterstellen können, allerdings kann insoweit keineswegs das Einverständnis des Versicherungsnehmers mit einem nicht in den Bedingungen verlautbarten Ausschluss des Terrorrisikos angenommen werden.

Dieses Ergebnis, wonach in der Regel das Terrorrisiko durch die Kriegsausschlussklausel nicht erfasst wird, ${ }^{92}$ entspricht auch der generellen Haltung des BGH, Risikoausschlüsse in Versicherungsverträgen eng auszulegen. ${ }^{93}$ Lediglich jene Ansicht, derzufolge es auf die Zurechnung zu einer Kriegspartei ankommt, führt aus der maßgeblichen Sicht eines verständigen Versicherungsnehmers zu einem Risikoausschluss, sofern der Terrorakt einer Kriegspartei zurechenbar ist. Dabei dürfte es sich freilich praktisch gesehen um Ausnahmefälle handeln. Jedenfalls bei sog. Massenrisiken ist Terror hingegen regelmäßig, sofern dies nicht ausdrücklich vereinbart ist, dem Krieg nicht gleichzustellen; ${ }^{4}$ für Großrisiken i. S. von Art. 10 Abs. 1 EGVVG gilt im Grundsatz nichts anderes.

Die genannten Parallelen zwischen Terror und Krieg haben jedoch für die künftige Klauselgestaltung Bedeutung. ${ }^{95}$ Da ebenso wie bei den Kriegsklauseln die schwere Kalkulierbarkeit des Risikos und die möglicherweise exorbitante Schadenshöhe eine Deckung erschweren, kann ein Ausschluss des Terrorrisikos etwa für den Fall vorgesehen werden, dass ein bestimmter Mindestschaden eintritt. Alternativ lässt sich das vom Versicherer übernommene Risiko dadurch begrenzen, dass die Versicherungssumme nicht zu hoch angesetzt und eine jährliche Höchstentschädigung bei mehreren Schadensereignissen vereinbart wird (s. bereits oben III a.E.). ${ }^{96}$

Eine weitere Frage geht dahin, ob das Auftreten eines Terroraktes als objektive Gefahrerhöhung i. S. des $\$ 27$ VVG anzusehen ist. Dies würde dem Versicherer eine Kündigung nach $\S 27$ Abs. 1 VVG ermöglichen und könnte nach Maßgabe des $\S 28$ VVG sogar zur Leistungsfreiheit führen. Das LG München $\mathrm{I}^{97}$ hat die Frage hinsichtlich der Anschläge vom 11.9.2001 verneint, wofür sich gute Gründe anführen lassen; die Frage kann hier freilich nicht vertieft werden.

92 So etwa auch R. M. Beckmann, ZIP 2002, 1125, 1127; Präve, in: Beckmann/MatuscheBeckmann (Fn. 26), § 10 Rn. 209; Schroeder, Die Kriegsgefahr im deutschen Versicherungsrecht, 1996, S. $120 \mathrm{f}$.

93 S. nur BGH VersR 2003, 360. Zur Auslegung von AVB allg. Prölss, in: Prölss/Martin, VVG. 27. Aufl. 2004, Vorbem. III.

94 So im Erg. auch Dahlke, VersR 2003, 25, $30 \mathrm{f}$.

95 S. dazu auch Fricke, VersR 2002, 6, $10 \mathrm{f}$.

96 Dahlke, VersR 2003, 25, $32 \mathrm{f}$.

97 LG München I NVersZ 2001, 454 (betr. Veranstaltungs-Ausfallversicherung); so im Erg. auch R. M. Beckmann, ZIP 2002, 1125, 1127 ff. und tendenziell LG Köln RnS 2005, 345, 347. 
bb) Ineinandergreifen von Privatversicherung und Staatsdeckung

Ausgehend von dem Befund, dass es für die Träger größerer Risiken schwierig ist, Versicherungsschutz unter Einschluss des Terrorrisikos zu erlangen, ist in Deutschland im April 2002 ein Abkommen zwischen der Versicherungswirtschaft und dem Staat in der Art einer public-private-partnership geschlossen worden. Nach dessen ursprünglicher Fassung ist von Seiten der Privatversicherung eine Deckungskapazität von 3 Mrd. Euro zu beschaffen, die um eine Staatsgarantie im Umfang von weiteren $10 \mathrm{Mrd}$. Euro ergänzt wird. Zugänglich ist dieser Deckungsschutz für Risiken mit einem Versicherungswert von mehr als 25 Mio. Euro. Die jährliche Höchstentschädigungssumme ist auf 1,5 Mrd. Euro pro Versicherungsnehmer begrenzt. Erfasst werden nur auf deutschem Territorium eintretende Schäden. Terror wird in Lit. A 1 Nr. 2 der Allgemeinen Bedingungen für die Terrorversicherung (ATB) wie folgt definiert: „Terrorakte sind jegliche Handlungen von Personen oder Personengruppen zur Erreichung politischer, religiöser, ethnischer oder ideologischer Ziele, die geeignet sind, Angst oder Schrecken in der Bevölkerung oder Teilen der Bevölkerung zu verbreiten und dadurch auf eine Regierung oder staatliche Einrichtungen Einfluss zu nehmen." Die von der Versicherungswirtschaft zu stellende Kapazität von $3 \mathrm{Mrd}$. Euro beruht auf dem Szenario eines Anschlags auf das Bankenviertel von Frankfurt/Main und stellt den mutmaßlichen Höchstschaden eines in Deutschland stattfindenden Terroraktes dar. ${ }^{98}$ Zur Deckung jener Kapazität ist die Extremus $A G$ gegründet worden, die als auf Terrorrisiken spezialisierter Erst- und teils auch Rückversicherer am Markt auftritt. Mittlerweile ist der Umfang reduziert worden. Die von der Privatwirtschaft aufzubringende Summe von nunmehr 2 Mrd. Euro teilen sich deutsche Erstversicherer und internationale Rückversicherer ${ }^{99}$. Die hinzukommende Staatsgarantie von inzwischen 8 Mrd. Euro hat das Bundesfinanzministerium erst jüngst bis zum Jahresende 2007 verlängert.

Das Engagement des Staates kommt nicht von ungefähr. Zwar kann er im Gegenzug für die von ihm gegebene Garantie einen (vergleichsweise geringen) Teil der vereinnahmten Prämien beanspruchen. ${ }^{100}$ Dies ist jedoch nicht der Beweggrund dafür, dass der Staat sich hier einschaltet, während die Deregulierung der europäischen Versicherungsmärkte in den 1990er Jahren und das damit einhergehende Ende der öffentlichrechtlichen Feuerversicherungsmonopole ${ }^{101}$ wirtschaftspolitisch gerade in die Gegenrichtung gewiesen haben. Entscheidend ist vielmehr Folgendes: Käme kein privatwirtschaftlich organisiertes Ausgleichssystem für Terrorschäden auf, so würde deren Behebung vielfach auf dem Staat lasten. Zwar bestehen regelmäßig keine Zahlungsansprüche der Geschädigten aus Amtshaftung (§ 839 BGB i.V. mit Art. 34 S. 1 GG), da es typischerweise an einer schuldhaften Amtspflichtverletzung fehlt. ${ }^{102}$ Politisch wird jedoch der Staat wegen der gesamtwirtschaftlichen Bedeutung der inneren und äuße-

98 Wolgast, in: Thomann/Graf von der Schulenburg (Hrsg.), War, Terrorism and Insurance in Europe after September 11, 2001, 2004, S. 53, 75 mit Fn. 47.

99 Wolgast (Fn. 98), S. 53, 76. Zum Deckungskonzept instruktiv Langheid/Rupietta, NJW 2005, 3233, $3234 \mathrm{ff}$.

100 Wolgast (Fn. 98), S. 53, 76.

101 S. dazu W.-H. Roth, in: Festschrift für Steindorff, 1990, S. $1313 \mathrm{ff}$.

102 Vgl. auch Dimski, VersR 1999, 804, 809 ff. (zu Tumultschäden). 
ren Sicherheit sowie aufgrund seiner allgemeinen Fürsorgepflicht in der Verantwortung gesehen. ${ }^{103}$ Katastrophenschutz ist nicht allein hinsichtlich der Vermeidung und der technischen Bewältigung von Katastrophen eine öffentliche Aufgabe (vgl. Art. 35 Abs. 2, 3 GG) ${ }^{104}$, sondern in gewissem Umfang auch in Bezug auf den Ausgleich ihrer wirtschaftlichen Folgen. Dies gilt bei Terroranschlägen deshalb in besonderem Maße, weil sie in der Regel gezielt gegen das Gemeinwesen gerichtet sind. Auch wenn es zu weit geht, hieraus abzuleiten, dass es nicht richtig sei, Terrorschäden überhaupt mit Mitteln der Privatwirtschaft abzudecken, sondern dass sich der Staat generell zur Wiedergutmachung von Terrorschäden bereit finden müsse ${ }^{105}$, besteht ein Interesse der Allgemeinheit daran, dass Terrorrisiken gedeckt werden. Der Staat handelt daher innerhalb seiner Kompetenzen, wenn er sich an einem solchen Deckungsmodell beteiligt.

Auch ausländische Staaten haben sich in die Deckung von Terrorrisiken eingeschaltet. So sind in einer ganzen Reihe von Ländern gesetzgeberische Maßnahmen getroffen worden, um eine Versicherungsdeckung für Terrorschäden zu erreichen. ${ }^{106}$ Zudem engagieren sich etwa Frankreich und das Vereinigte Königreich gleichfalls finanziell in Gestalt einer Staatsgarantie ${ }^{107}$.

\section{Fazit}

Katastrophen stellen die Leistungsfähigkeit der Privatversicherung auf eine harte Probe. Sie können - anders als sonstige Risiken - nicht nur für den originären Risikoträger (Versicherungsnehmer), sondern auch den Versicherer existenzbedrohende Folgen haben. Wie die obigen Ausführungen gezeigt haben, bestehen eine ganze Reihe von Handlungsmöglichkeiten. So kann das Risiko wegen der bestehenden Kalkulationsschwierigkeiten gänzlich ausgeschlossen werden. Dieser Ausweg hat freilich etwas Resignatives. Er widerspricht auch einem keineswegs selten anzutreffenden Bedürfnis nach möglichst weit reichender Absicherung auch gegen nicht alltägliche Risiken. Ein auch volkswirtschaftlich nutzbringender Mittelweg kann darin liegen, dass Versicherungsschutz gewährt wird, dabei aber Schutzvorkehrungen ausbedungen und angemessene Höchstentschädigungssummen festgesetzt werden. Deckt der Versicherer das Risiko eingeschränkt oder in vollem Umfang, so kann er es zudem zumindest zum Teil

103 S. etwa U. Hübner, ZVersWiss 1981, 1, 13 ff.; dens. (Fn. 76), S. 179, 187 f.; Jannott/Glotzmann, in: FS Hauß, 1978, S. 121 ff.; Wolgast, ifo-Schnelldienst 2001, 12, 13 ; vgl. auch A. Bruns, JZ 2005, 13, 14; Thomann, Pool Re: Versicherung von Terrorrisiken in Großbritannien, 2003, S. 1 ; ferner D. Kessler, in: Rapport fait au nom de la Commission d'enquête sur la sûreté des installations industrielles et de centres de recherche et sur la protection des personnes et de l'environnement en cas d'accident industriel majeur, Assemblée Nationale, No. 3559, Tome II, p. 185. S. auch allg. Limbach, Ist die kollektive Sicherheit der Feind der individuellen Freiheit?, 2002, S. 5.

104 S. dazu den Beitrag von Trute in diesem Heft.

105 Dafür Rohde-Liebenau, ZfV 2002, 71; dagegen zutr. Dahlke, VersR 2003, 25, 27.

106 Vgl. die Übersichten bei A. Bruns, JZ 2005, 13, 15 ff.; Hübner (Fn. 76), S. 179, 188 ff.; Thomann (Fn. 103), S. 42 ff., 82 ff.; Wolgast (Fn. 98), S. 53, 82 f.

107 S. dazu den Überblick bei Thomann (Fn. 103), S. 82 f. 
im Wege der Mit- oder Rückversicherung oder am Kapitalmarkt an Dritte weiterreichen. Gerade die neuen, speziell auf Katastrophenrisiken zugeschnittenen Kapitalmarktinstrumente zeigen, dass zur Bewältigung dieser außergewöhnlichen Risiken bisweilen auch unkonventionelle Wege beschritten werden. Bietet der Versicherer eine Deckung an, so ist es am Risikoträger zu entscheiden, ob er hierauf eingehen und dafür die geforderte Prämie aufwenden soll. Die Antwort hierauf wird ihm aus eben jenen Gründen, die Katastrophenrisiken für den Versicherer so schwer kalkulierbar machen, bisweilen deutlich weniger leicht fallen als bei alltäglichen Standardrisiken. 